\title{
Obtenção de ácidos graxos por cristalização do óleo de pescado fracionado por hidrólise enzimática
}

\author{
Fatty acid isolation by crystallization of fish oil fractioned by enzymatic hydrolysis
}

\author{
Márcia Elisa da Silva PADILHA, Walter AUGUSTO-RUIZ ${ }^{1 \star}$
}

\begin{abstract}
Resumo
Este trabalho teve por objetivo estabelecer um processo para obter e caracterizar os produtos da cristalização das frações do óleo de pescado hidrolisado com lipase pancreática. A hidrólise enzimática foi realizada em 60 minutos com uma concentração de substrato de $1262 \mu$ mols, $38^{\circ} \mathrm{C}$, pH 8 e com uma concentração de extrato enzimático de 7,647 mg.mL $\mathrm{m}^{-1}$. Os produtos da hidrólise foram separados por cromatografia em coluna e caracterizados por cromatografia em camada delgada e cromatografia em fase gasosa. A temperatura de cristalização foi controlada entre $5^{\circ} \mathrm{Ce}-18^{\circ} \mathrm{C}$. A fração TAG foi melhor cristalizada a $0^{\circ} \mathrm{C}$ enquanto que as frações DAG + AGL e MAG foram cristalizadas a $5{ }^{\circ} \mathrm{C}$. Foi observado que o ácido docosahexaenoico (DHA) concentra-se na fase líquida da fração DAG + AGL representando $97,17 \%$ em relação ao óleo de pescado. O ácido palmítico foi cristalizado na fração DAG, perfazendo aproximadamente $50 \%$ do total de ácidos graxos. Foi observado que os ácidos graxos insaturados de cadeia longa, principalmente o EPA, concentram-se na fase sólida da fração TAG, esses resultados sugerem que o processo combinado de hidrólise enzimática seguido de cristalização possibilita a obtenção destes ácidos graxos do óleo de pescado.
\end{abstract}

Palavras-chave: cristalização de ácidos graxos; ácidos graxos poli-insaturados n-3; óleo de pescado; fracionamento por cristalização.

\begin{abstract}
The main objective of this work was to establish a process to characterize the crystallization products of fish oil fractions hydrolyzed with pancreatic lipase. Enzymatic hydrolysis was accomplished in a 60 minute hydrolysis employing a substrate concentration of $1262 \mathrm{mols}, 38^{\circ} \mathrm{C}$, with an enzyme concentration (protein) of $7.647 \mathrm{mg} \cdot \mathrm{mL}^{-1}, \mathrm{pH}$. The hydrolysis products were separated by column chromatography, thin layer chromatography, and gas chromatography. The crystallization temperature was controlled between $5^{\circ} \mathrm{C}$ and $-18^{\circ} \mathrm{C}$. The TAG fraction was best crystallized at $0{ }^{\circ} \mathrm{C}$ while the DAG + AGL and MAG fractions were crystallized at $5{ }^{\circ} \mathrm{C}$. It was observed that docosahexaenoic acid (DHA) concentrated in the liquid phase of the DAG + AGL fractions represented 97.17 per cent of the fish oil. Palmitic acid was crystallized in the DAG fraction and comprised about 50 per cent of the total fatty acids. The long chain polyunsaturated fatty acids, mainly the EPA, are concentrated in the solid phase of the TAG fraction. The results suggest that the isolation of fatty acids from fish oil can be accomplished using a combination of enzymatic hydrolysis followed by crystallization.
\end{abstract}

Keywords: crystallization of fatty acids; polyunsaturated fatty acids $n-3$; fish oil; fractionation by crystallization.

\section{Introdução}

Ácidos graxos n-3 são considerados essenciais com função importante no metabolismo celular. Através de estudos epidemiológicos tem sido demonstrado que os ácidos graxos poli-insaturados - PUFAs, principalmente o ácido eicosapentaenoico C 20:5n-3 (EPA) e o ácido docosahexaenoico C 22:6n3 (DHA), provenientes de óleos marinhos, são importantes na prevenção de doenças cardiovasculares, na prevenção e tratamento de doenças como hipertensão, câncer e artrite e outras desordens inflamatórias e autoimunes. $\mathrm{O}$ efeito benéfico dos PUFAs tem sido atribuído à diminuição da concentração dos triacilgliceróis séricos (TAG), à diminuição do colesterol, à redução da trombose, ao aumento da fluidez da membrana celular e de facilitar sua excreção e conversão em eicosanoides (KINSELLA, 1986; WANASUNDARA; SHAHIDI, 1998).
O conhecimento dos benefícios dos PUFAs leva a evidenciar mudança na dieta alimentar da sociedade ocidental, mencionase que a proporção do consumo de PUFAs a relação n- $6 / n-3$ de 1:1 tenha mudado de $1: 1$ para 10:1, respectivamente, ou ainda inclusive para 20-25:1 (SIMOPOULOS, 1991; HORROCKS; YEO, 1999). Portanto, o consumo de quantidades apropriadas de ácidos graxos n-6 e n-3 é recomendável. Tem sido sugerido que o consumo de concentrados de PUFAs, livre de ácidos graxos saturados, mostra melhor efeito que os próprios óleos marinhos, por permitir um baixo depósito diário de lipídios totais (WANASUNDARA; SHAHIDI, 1998; HORROCKS; YEO, 1999). A indústria farmacêutica e alimentícia tem usado diferentes métodos para preparar concentrados de PUFAs de óleos marinhos, entretanto, a maioria dos métodos produz concentrados de PUFAs na forma de mistura de ácidos graxos 
livres ou seus correspondentes ésteres alcalinos (AUGUSTO RUIZ; TORALLES, 1999; CUNHA; PINTO, 2002). Foi observado em animais de laboratório que os acilgliceróis são mais bem absorvidos que os respectivos ésteres alcalinos de ácidos graxos n-3. Também tem sido demonstrado que metil e etil ésteres de ácidos graxos insaturados hidrolisam a uma menor velocidade que seus correspondentes acilgliceróis. (WANASUNDARA; SHAHIDI, 1998).

Lipases microbianas têm sido utilizadas na hidrólise de óleos, na produção de concentrados de ácidos graxos n-3 na forma de acilgliceróis, na produção de óleos estruturados (YAMAGUCHI; AKOH; LAI, 2004) a partir de óleos marinhos em que se verificou a resistência destas lipases, as cadeias longas dos ácidos n-3 PUFAs (BOTTINO; VANDENBURG; REISER, 1967); entretanto, a hidrólise enzimática com lipases microbianas produziu maior quantidade de ácidos graxos livres na hidrólise do óleo de arroz (LOPEZ-MARTINEZ et al., 2006). A viscosidade do óleo é o fator mais importante na hidrólise enzimática quando se utiliza lipase microbiana (YU; BAHARIN, 2006).

A presença de duplas ligações cis de carbono-carbono em ácidos graxos resulta em dobras nas cadeias. Dessa forma, o grupo metil terminal dos ácidos graxos fecha-se em direção à ligação éster, a qual pode causar um efeito de obstáculo espacial para a ação das lipases. O alto efeito de dobra das cadeias de EPA e DHA é devido à presença de cinco e seis duplas ligações, respectivamente, aumentando o efeito do obstáculo espacial. Dessa forma, as lipases não podem alcançar a ligação entre estes ácidos graxos e o glicerol (SHIMADA et al., 1994; IKEMOTO; OTA, 1996; WANASUNDARA; SHAHIDI, 1998; NAMBOODIRI; CHATTOPADHYAYA, 2000).

Se for feito apenas o fracionamento de ácidos graxos livres ou seus mono ésteres, a separação será limitada somente pela eficiência dos métodos de fracionamento, sendo possível a separação quase quantitativa dos ácidos ou ésteres puros desde que seja empregada a metodologia adequada (RITNNER, 1996; CUNHA; PINTO, 2002).

Separar e identificar ácidos graxos do óleo de pescado fracionado através de processo de hidrólise enzimática foi o principal objetivo deste trabalho.

\section{Material e métodos}

\subsection{Fracionamento enzimático do óleo de pescado}

O óleo de pescado foi obtido em empresa da cidade do Rio Grande, RS e hidrolisado conforme fluxograma da Figura 1, utilizando lipase pancreática porcina (SIGMA). O grau de hidrólise foi determinado segundo Padilha (2002), as frações de triacilglicerídeos, diacilglicerídeos, monoacilglicerídeos e ácidos graxos foram separadas por cromatografia em coluna e cromatografia em camada delgada (PADILHA, 2002; STAUFFERT, 2000).

As frações coletadas foram diluídas em hexano p.a. até atingir concentração de $1 \mathrm{mg} \cdot \mu \mathrm{L}^{-1}$ (PADILHA, 2002), acondicionadas ao amparo da luz e estocadas em congelamento até sua caracterização.

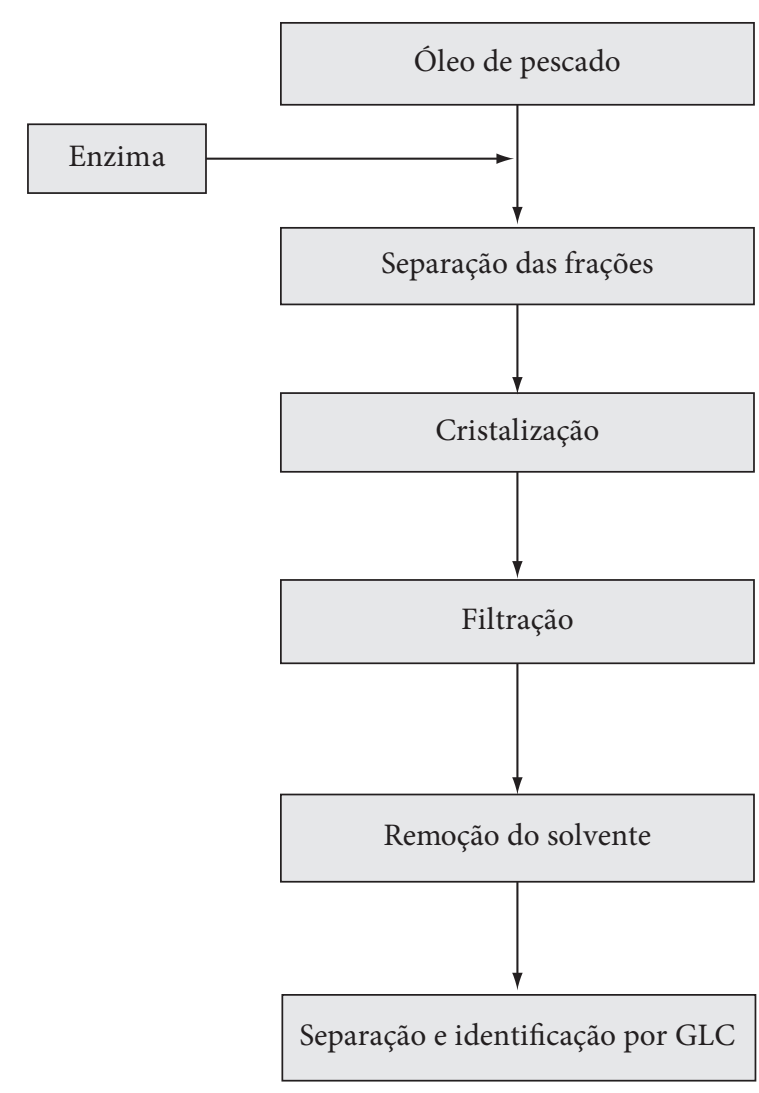

Figura 1. Diagrama do processo de cristalização e caracterização das frações do óleo de pescado.

A cristalização foi realizada por 60 minutos à temperatura entre $0 \mathrm{a}-18^{\circ} \mathrm{C}$, controlada através de termostato eletrônico. A fração TAGs (triacilglicerídeos) foi cristalizada a $0{ }^{\circ} \mathrm{C}$, enquanto que as frações DAG + AGL (diacilglicerídeos mais ácidos graxos livres) e MAG (monoacilglicerídeos) foram cristalizadas a $-5^{\circ} \mathrm{C}$.

Os sólidos foram separados por filtração à mesma temperatura de cristalização, em aproximadamente 30 minutos. O solvente foi removido à temperatura ambiente sob atmosfera de nitrogênio. Sólidos e sobrenadantes foram estocados a $-18^{\circ} \mathrm{C}$ em ambiente de nitrogênio até sua caracterização.

\subsection{Determinação de ácidos graxos}

A concentração dos ácidos graxos foi determinada por cromatografia em fase gasosa (MAIA, 1992), cromatógrafo Varian-3400 CX, detector de ionização de chama (FID), coluna capilar com fase estacionária de ácido nitrotereftálico modificado por polietileno glicol, com $30 \mathrm{~m}$ de comprimento e 0,545 $\mathrm{mm}$ de diâmetro com um filme de $1 \mathrm{~mm}$ de espessura. $O$ gás de arraste foi o nitrogênio a $2 \mathrm{~mL} / \mathrm{min}$. As temperaturas do injetor e do detector foram de 250 e $270{ }^{\circ} \mathrm{C}$, respectivamente. A temperatura inicial da coluna foi de $160^{\circ} \mathrm{C}$, permanecendo nesta temperatura por 1 minuto. A seguir, foram realizadas duas programações de temperatura: aumento a $2{ }^{\circ} \mathrm{C} / \mathrm{min}$ até $200^{\circ} \mathrm{C}$, permanecendo por 2 minutos; aumento a $20^{\circ} \mathrm{C} / \mathrm{min}$ até 
$220^{\circ} \mathrm{C}$, permanecendo nesta temperatura durante 40 minutos, totalizando 64 minutos.

A quantidade de amostra injetada foi de $1 \mu \mathrm{L}$. Os ácidos graxos foram identificados pela comparação direta dos tempos de retenção com padrões Sigma, Supelco e quantificados por normalização de áreas, através do software Varian Star 4.51.

\section{Resultados e discussão}

\subsection{Grau de hidrólise}

O maior grau de hidrólise $(46 \% \pm 2 \%)$ foi atingido nas seguintes condições: 60 minutos de reação, $38^{\circ} \mathrm{C}, \mathrm{pH} 8,0$ tampão $\mathrm{NH}_{4} \mathrm{Cl}-\mathrm{NH}_{4} \mathrm{OH}, 1259,35 \mu$ mols de substrato e 7,65 mg.mL $\mathrm{mL}^{-1}$ do extrato enzimático.

\subsection{Perfil cromatográfico de ácidos graxos}

O perfil dos ácidos graxos do óleo de pescado (Tabela $1 \mathrm{e}$ Figura 2) mostra um total de 69\% de ácidos graxos insaturados, o que está de acordo com outros autores (RITTNER, 1996; CONTRERAS, 1994), entretanto a alta concentração de ácido palmítico pode ser indicativa de tratar-se de um óleo de pescado não purificado (RITTNER, 1996). Os ácidos graxos eicosanopentaenoico 20:5 (EPA) e docosanohexaenoico 22:6 (DHA) perfazem em torno de $21 \%$ do total. É interessante verificar a presença de uma grande quantidade de ácidos graxos insaturados o que lhe confere um potencial muito importante para sua separação.

Tabela 1. Composição de ácidos graxos do óleo de pescado.

\begin{tabular}{rrrr}
\hline $\mathrm{AG}^{\star}$ & \multicolumn{1}{c}{$\%$} & $\mathrm{AG}$ & \multicolumn{1}{c}{$\%$} \\
\hline $14: 0$ & $4,60 \pm 0,16$ & $20: 0$ & $2,87 \pm 0,03$ \\
$16: 0$ & $16,89 \pm 0,10$ & $20: 1$ & $5,36 \pm 0,02$ \\
$16: 1$ & $7,11 \pm 0,19$ & $20: 3$ & $0,99 \pm 0,01$ \\
$18: 0$ & $2,23 \pm 0,04$ & $20: 4$ & $6,87 \pm 0,01$ \\
$18: 1$ & $19,28 \pm 0,11$ & $20: 5$ & $4,82 \pm 0,01$ \\
$18: 2$ & $3,55 \pm 0,06$ & $22: 5$ & $0,99 \pm 0,01$ \\
$18: 3$ & $1,22 \pm 0,00$ & $22: 6$ & $16,27 \pm 0,22$ \\
\hline
\end{tabular}

*aproximadamente 6,95\% de ácidos graxos não foram identificados; $A G$ = ácido graxo; $\mathrm{e}(\mathrm{m} \pm \mathrm{s})=$ média \pm desvio padrão.

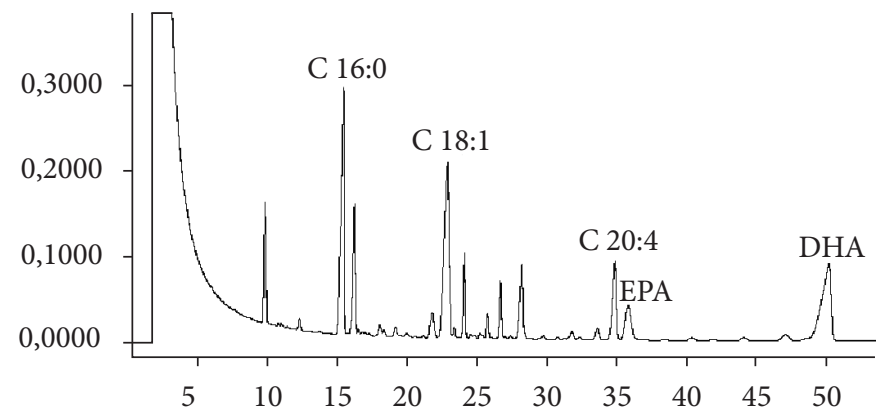

Figura 2. Perfil cromatográfico de ácidos graxos do óleo de pescado.
O óleo de pescado geralmente é obtido por processo hidrotérmico a partir de espécies pelágicas. Portanto sua composição varia segundo as espécies e, inclusive, segundo a estação do ano, sexo, ciclo reprodutor, dentre outros fatores (BERNARDINI, 1986; GONÇALVES; SOUZA-SOARES, 2000, 1998; WINDSOR; BARLOW, 1996). Os peixes pelágicos, por sua condição de habitação e por formar grandes cardumes, são de grande importância industrial (CONTRERAS, 1994). Segundo alguns autores (GONÇALVES; SOUZA-SOARES, 2000, 1998), as espécies pelágicas parecem ser as melhores fontes de EPA e DHA. Esse processo permite a separação de proteínas de outros materiais, como os ácidos graxos livres e água, considerados impurezas, que são eliminados em processos como degomagem, refino, branqueamento ou descoloração, desodorização e fracionamento (RITTNER, 1996; MORAES, 2000; MORAES; SOUZA-SOARES, 2000; ZILLER, 1986).

\subsection{Composição das frações do hidrolisado enzimático do óleo de pescado}

A fração AGL representa aproximadamente 56\% dos produtos da hidrólise do óleo de pescado (Tabela 2) e difere significativamente das frações MAG, DAG e TAG, sendo que a fração TAG está em menor concentração como resultado da hidrólise enzimática.O peso molecular dos MAG foi calculado com base na mono-oleína (356,50 g. $\left.\mathrm{mol}^{-1}\right)$, o dos DAG com base na Di-oleína $\left(621,00\right.$ g.mol $\left.{ }^{-1}\right)$, o dos TAG com base na Tri-oleína $\left(885,40\right.$ g.mol $\left.{ }^{-1}\right)$ e o dos ácidos graxos com base no ácido oleico $\left(282,00 \mathrm{~g} \cdot \mathrm{mol}^{-1}\right)$.

A concentração do ácido palmítico diminui nas frações AGL e DAG, entretanto aumenta na fração MAG. Vários autores relatam maior incidência do ácido palmítico na posição n2 de triacilglicerídeos; nessa posição a lipase tem demonstrado menor atividade (XU et al., 1998a, b; ANDO; SATAQUI; TAKAHASHI, 2000) e relata-se que a atividade da lipase é maior quando tem como substrato o ácido oleico do que o ácido palmítico (TORALLES, 1998).

Na Tabela 3 e Figuras 3 e 4, verifica-se que o ácido palmítico (C16:0) perfaz aproximadamente 48,75\% da fase sólida da fração MAG, o que representa aproximadamente 3 vezes a concentração original no óleo de pescado, portanto, este ácido graxo pode ser separado do óleo de pescado na cristalização da fração MAG. Reporta-se que a cristalização lenta promove cristais homogêneos porém a temperatura e o tempo de cristalização são as variáveis mais importantes na formação do cristal (MISKANDAR et al., 2006). A fase líquida da cristalização da fração MAG apresenta aproximadamente duas vezes a concentração do ácido docosahexenoico (C22:6) originalmente encontrado no óleo de pescado, indicando que é uma possibilidade para sua separação e purificação. Resultado similar foi encontrado na fase líquida das frações do óleo de arroz cristalizado a baixas temperaturas (YU et al. 2006).

A Tabela 4 permite verificar que a fase sólida da cristalização da fração DAG + AGL é constituída principalmente pelos graxos saturados mirístico C14:0 e palmítico C16:0, perfazendo aproximadamente $50 \%$ do total de ácidos graxos solidificados, no entanto também apresenta aproximadamente 15\% de ácido 
Tabela 2. Composição dos produtos de hidrólise enzimática do óleo de pescado.

\begin{tabular}{lcccc}
\hline Fração & Massa $(\mathrm{g})$ & \multicolumn{1}{c}{$\mu$} & \multicolumn{1}{c}{$\mu$ mols $^{*}$} & \\
\hline MAG & $252,66 \pm 12,23$ & $22,29 \pm 0,91$ & $708,72 \pm 34,31$ & $23,31 \pm 1,09$ \\
DAG & $227,53 \pm 2,47$ & $20,07 \pm 0,07$ & $366,39 \pm 3,96$ & $12,05 \pm 0,11$ \\
TAG & $184,34 \pm 10,86$ & $16,26 \pm 0,84$ & $208,19 \pm 12,27$ & $7,07 \pm 0,44$ \\
AGL & $468,66 \pm 17,01$ & $41,38 \pm 1,82$ & $1661,91 \pm 60,31$ & $56,42 \pm 1,86$ \\
Total & $1133,19 \pm 8,55$ & 100,00 & $2945,22 \pm 9,76$ & 100,00 \\
\hline
\end{tabular}

$\mathrm{MAG}=$ monoacilglicerídeos; $\mathrm{DAG}=$ diacilglicerídeos; $\mathrm{TAG}=$ triacilglicerídeos; $\mathrm{AGL}=$ ácidos graxos livres; e $\mathrm{m} \pm \mathrm{s}=\mathrm{média} \pm$ desvio-padrão $(\mathrm{n}=3)$.

Tabela 3. Teor de ácidos graxos (\%) das fases líquida e sólida da cristalização da fração monoacilglicerídica.

\begin{tabular}{|c|c|c|c|c|c|}
\hline $\mathrm{AG}$ & Óleo & FL & $\%^{*}$ & FS & $\%^{*}$ \\
\hline $14: 0$ & $4,60 \pm 0,16$ & $5,83 \pm 0,39$ & $+26,74$ & $12,61 \pm 0,11$ & $+174,13$ \\
\hline $16: 0$ & $16,89 \pm 0,10$ & $8,21 \pm 0,25$ & $-105,72$ & $48,75 \pm 0,33$ & $+188,63$ \\
\hline $16: 1$ & $7,11 \pm 0,19$ & $6,06 \pm 0,12$ & $-17,33$ & $4,29 \pm 0,01$ & $-65,73$ \\
\hline $18: 0$ & $2,23 \pm 0,04$ & n.q. & - & $2,36 \pm 0,07$ & $+5,83$ \\
\hline $18: 1$ & $19,28 \pm 0,11$ & $13,79 \pm 0,30$ & $-39,81$ & $8,69 \pm 0,20$ & $-121,86$ \\
\hline $18: 2$ & $3,55 \pm 0,06$ & $3,59 \pm 0,09$ & $+1,13$ & $2,03 \pm 0,03$ & $-74,88$ \\
\hline $18: 3$ & $1,22 \pm 0,00$ & n.q. & - & n.q. & - \\
\hline $20: 0$ & $2,87 \pm 0,03$ & n.q. & - & $1,27 \pm 0,06$ & $-125,98$ \\
\hline $20: 1$ & $5,36 \pm 0,02$ & $2,59 \pm 0,12$ & $-106,95$ & $1,35 \pm 0,08$ & $-297,03$ \\
\hline $20: 3$ & $0,99 \pm 0,01$ & n.q. & - & n.q. & - \\
\hline $20: 4$ & $6,87 \pm 0,01$ & $6,66 \pm 0,18$ & $-3,15$ & $2,38 \pm 0,03$ & $-141,05$ \\
\hline $20: 5$ & $4,82 \pm 0,01$ & $2,52 \pm 0,02$ & $-91,27$ & $1,08 \pm 0,01$ & $-359,05$ \\
\hline $22: 5$ & $0,99 \pm 0,01$ & n.q. & - & n.q. & - \\
\hline $22: 6$ & $16,27 \pm 0,22$ & $32,08 \pm 0,18$ & $+97,17$ & $9,80 \pm 0,19$ & $-86,02$ \\
\hline
\end{tabular}

$\mathrm{AG}=$ ácido graxo, $\mathrm{m} \pm \mathrm{s}(\mathrm{n}=3)$ = média \pm desvio padrão; *percentual de variação com referência ao óleo de pescado; $\mathrm{FL}$ = fração líquida; $\mathrm{FS}=$ fração sólida; e n.q. = não quantificados.

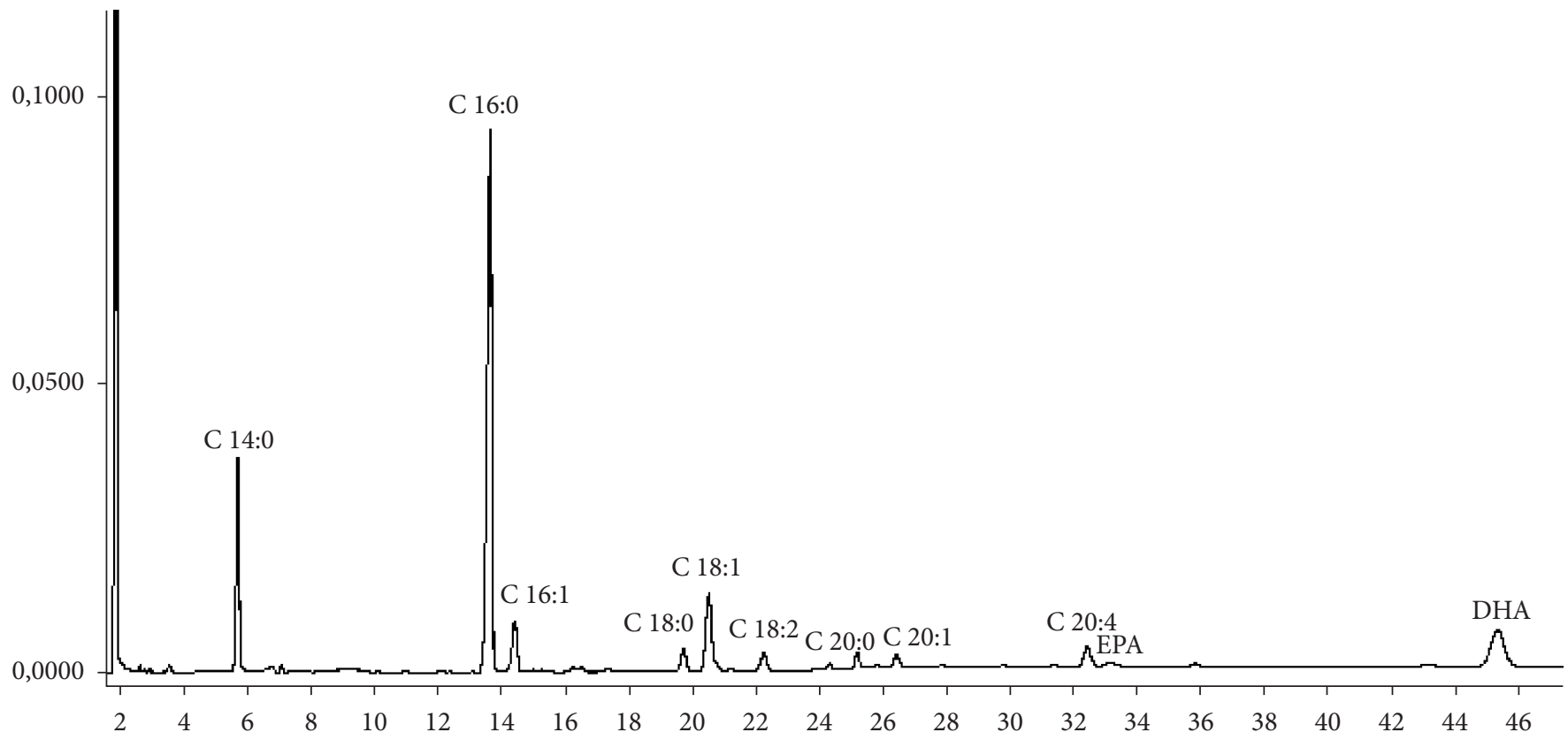

Figura 3. Ácidos graxos da fase sólida da cristalização da fração monacilglicerídica.

oleico (C18:1). Estes ácidos têm propriedades físicas diferentes, o que dificulta a cristalização da fração DAG e AGL, e têm sido considerados interferentes do processo de fracionamento através da cristalização (RITTNER, 1996; ZILLER, 1996).
Na fase líquida da cristalização da fração DAG + AGL, a concentração do ácido oleico C18:1 perfaz aproximadamente $25,20 \%$, concentração essa três vezes maior que no óleo de pescado. Este resultado sugere que a lipase pancreática porcina 


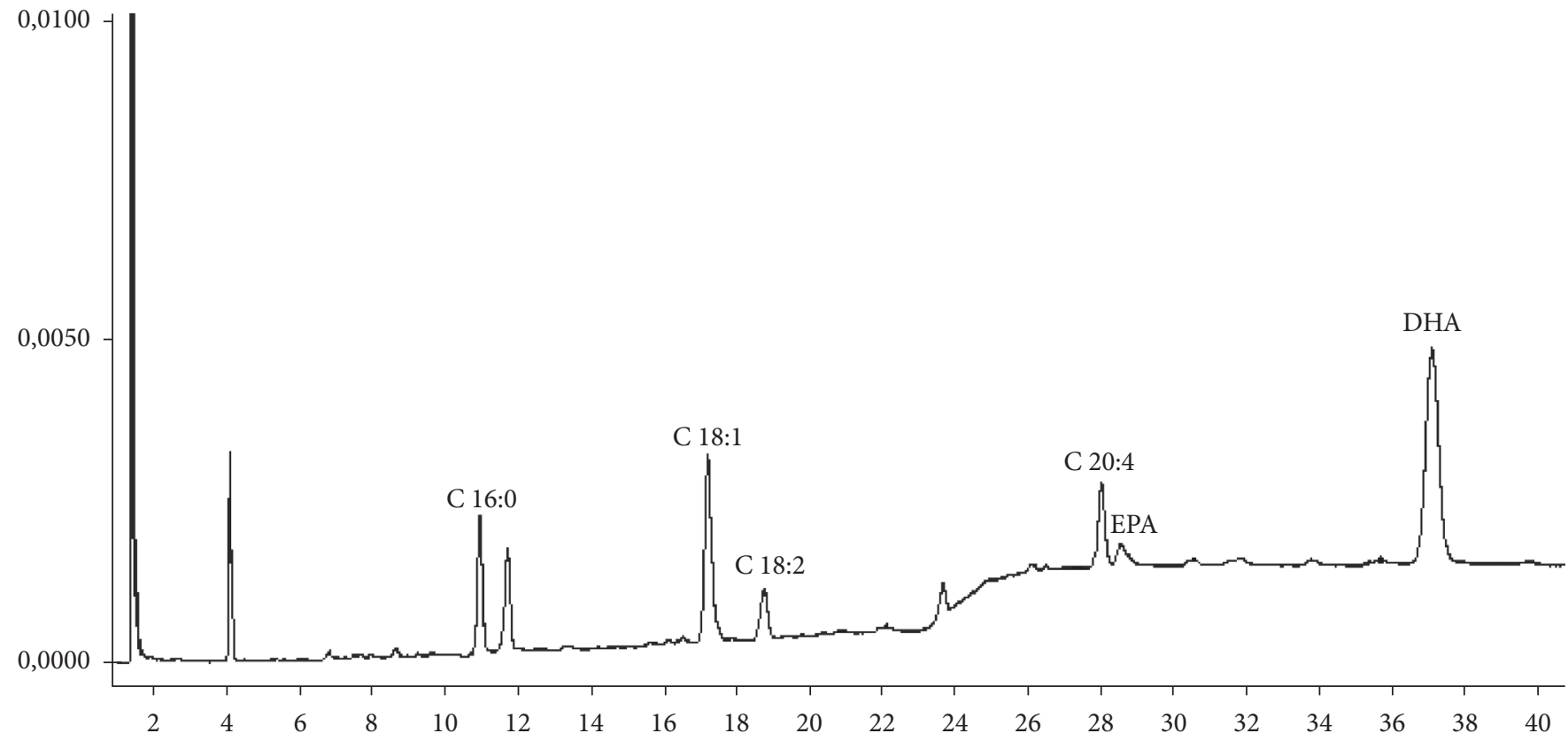

Figura 4. Ácidos graxos da fase líquida da cristalização da fração monoacilglicerídica.

Tabela 4. Composição percentual de ácidos graxos das fases líquida e sólida da cristalização da fração diacilglicerídica + ácidos graxos livres.

\begin{tabular}{|c|c|c|c|c|c|}
\hline AG & Óleo & FL & $\%^{*}$ & FS & $\%^{*}$ \\
\hline $14: 0$ & $4,60 \pm 0,16$ & $8,09 \pm 0,09$ & $+75,87$ & $10,09 \pm 0,10$ & $+119,34$ \\
\hline $16: 0$ & $16,89 \pm 0,10$ & $20,08 \pm 0,08$ & $+18,89$ & $39,92 \pm 0,06$ & $+136,35$ \\
\hline $16: 1$ & $7,11 \pm 0,19$ & $11,25 \pm 0,10$ & $+58,23$ & $8,44 \pm 0,13$ & $+18,70$ \\
\hline $18: 0$ & $2,23 \pm 0,04$ & $2,61 \pm 0,06$ & $+17,04$ & $3,27 \pm 0,10$ & $+46,64$ \\
\hline $18: 1$ & $19,28 \pm 0,11$ & $25,20 \pm 0,10$ & $+30,70$ & $14,68 \pm 0,10$ & $-31,33$ \\
\hline $18: 2$ & $3,55 \pm 0,06$ & $4,43 \pm 0,10$ & $+24,79$ & $2,71 \pm 0,06$ & $-30,09$ \\
\hline $18: 3$ & $1,22 \pm 0,00$ & - & - & $1,16 \pm 0,02$ & $-5,17$ \\
\hline $20: 0$ & $2,87 \pm 0,03$ & $3,15 \pm 0,05$ & $+9,76$ & $2,51 \pm 0,03$ & $-14,34$ \\
\hline $20: 1$ & $5,36 \pm 0,02$ & $5,63 \pm 0,07$ & $+5,04$ & $2,87 \pm 0,02$ & $-86,76$ \\
\hline $20: 3$ & $0,99 \pm 0,01$ & - & - & - & - \\
\hline $20: 4$ & $6,87 \pm 0,01$ & $6,26 \pm 0,01$ & $-9,74$ & $4,32 \pm 0,02$ & $-59,03$ \\
\hline $20: 5$ & $4,82 \pm 0,01$ & $4,15 \pm 0,03$ & $-17,35$ & $2,03 \pm 0,03$ & $-137,44$ \\
\hline $22: 5$ & $0,99 \pm 0,01$ & - & - & $0,77 \pm 0,00$ & $-28,57$ \\
\hline $22: 6$ & $16,27 \pm 0,22$ & $9,13 \pm 0,08$ & $-78,20$ & $6,17 \pm 0,19$ & $-163,69$ \\
\hline
\end{tabular}

AG = ácidos graxos; $\mathrm{FL}$ = fração líquida; $\mathrm{FS}$ = fração sólida; $(\mathrm{m} \pm \mathrm{s})$ = média \pm desvio padrão $(\mathrm{n}=3) ; \mathrm{e}^{\star}$ percentual de variação.

atua liberando maior quantidade de ácido oleico e que este se encontra na posição n1 ou n2 no TAG. Os resultados sugerem que a cristalização da fração DAG + AGL favorece a separação de ácidos graxos livres.

Em relação aos ácidos graxos insaturados de cadeia longa, pode-se observar que o melhor resultado para o EPA (ácido eicosapentaenoico C20:5) foi a fase sólida da fração triacilglicerídica (Tabela 5). Este valor pode ser explicado pelo fato do EPA ser resistente à ação da lipase pancreática porcina, pois apresenta uma configuração que gera um obstáculo espacial à sua ação, devido à presença das duplas ligações, como descrito no mecanismo proposto por Bottino, Vandenburg e Reiser (1967). O fato de apresentar melhor concentração na fase sólida, apesar das duplas ligações abaixarem consideravelmente o seu ponto de fusão, deve ser por encontrar-se na fração dos TAG, ou seja, os resultados sugerem que o EPA estava associado na mesma molécula triacilglicerídica com dois outros ácidos graxos de maior ponto de fusão e que suas características prevaleceram em relação às características do EPA, uma vez que se sabe que os triacilglicerídeos tendem a assumir as características médias dos ácidos graxos, já que três diferentes ácidos, de distinta natureza e propriedades, podem estar combinados numa mesma molécula triacilglicerídica. 
Tabela 5. Composição de ácidos graxos (\%) das fases líquida (FL) e sólida (FS) da cristalização da fração triacilglicerídica.

\begin{tabular}{|c|c|c|c|c|c|}
\hline $\mathrm{AG}$ & Óleo & FL & $\%$ & FS & $\%$ \\
\hline $14: 0$ & $4,60 \pm 0,16$ & $5,96 \pm 0,09$ & $+29,56$ & $4,29 \pm 0,15$ & $-7,23$ \\
\hline $16: 0$ & $16,89 \pm 0,10$ & $16,52 \pm 0,10$ & $-2,24$ & $15,96 \pm 0,03$ & $+13,68$ \\
\hline $16: 1$ & $7,11 \pm 0,19$ & $10,39 \pm 0,22$ & $+46,13$ & $8,93 \pm 0,03$ & $+6,46$ \\
\hline $18: 0$ & $2,23 \pm 0,04$ & $2,06 \pm 0,07$ & $-8,25$ & $2,77 \pm 0,01$ & $+24,21$ \\
\hline $18: 1$ & $19,28 \pm 0,11$ & $25,12 \pm 0,32$ & $+30,29$ & $20,88 \pm 0,23$ & $+8,30$ \\
\hline $18: 2$ & $3,55 \pm 0,06$ & $3,96 \pm 0,10$ & $+11,55$ & $3,48 \pm 0,10$ & $+2,01$ \\
\hline $18: 3$ & $1,22 \pm 0,00$ & $1,46 \pm 0,10$ & $+19,67$ & $1,17 \pm 0,01$ & $-4,27$ \\
\hline $20: 0$ & $2,87 \pm 0,03$ & $3,47 \pm 0,10$ & $+20,90$ & $2,58 \pm 0,07$ & $-11,24$ \\
\hline $20: 1$ & $5,36 \pm 0,02$ & $6,39 \pm 0,15$ & $+19,21$ & $5,82 \pm 0,08$ & $+8,58$ \\
\hline $20: 3$ & $0,99 \pm 0,01$ & $1,03 \pm 0,00$ & $+4,04$ & $1,02 \pm 0,01$ & $+3,03$ \\
\hline $20: 4$ & $6,87 \pm 0,01$ & $6,49 \pm 0,18$ & $-5,85$ & $6,29 \pm 0,06$ & $-9,22$ \\
\hline $20: 5$ & $4,82 \pm 0,01$ & $4,77 \pm 0,02$ & $-1,05$ & $5,20 \pm 0,03$ & $+7,88$ \\
\hline $22: 5$ & $0,99 \pm 0,01$ & $0,97 \pm 0,01$ & $+2,02$ & $0,71 \pm 0,00$ & $-39,44$ \\
\hline $22: 6$ & $16,27 \pm 0,22$ & $10,28 \pm 0,18$ & $-58,27$ & $12,75 \pm 0,25$ & $-27,61$ \\
\hline
\end{tabular}

$\mathrm{AG}=$ ácido graxo; $\mathrm{e}(\mathrm{m} \pm \mathrm{s})=$ média \pm desvio padrão $(\mathrm{n}=3)$.

\section{Conclusão}

O óleo de pescado apresentou um teor de $21 \%$ de ácidos graxos poli-insaturados - PUFAs, como eicosanopentaenoico e docosanohexaenoico, confirmando seu potencial para a obtenção dos PUFAs.

O grau de hidrólise pode ser um indicativo da extensão da hidrólise enzimática, assim, um valor de aproximadamente $46 \%$ de grau de hidrólise indica que o óleo de pescado é um bom substrato para a lipase pancreática porcina: o fracionamento enzimático produziu as frações MAG, DAG AGL e TAG.

As frações do óleo de pescado apresentam composição de ácidos graxos diferente. $\mathrm{O}$ ácido palmítico pode ser separado por cristalização da fração MAG, o ácido oleico pode ser separado na fração DAG + AGL; os ácidos graxos poli-insaturados, principalmente EPA, podem ser separados da fração TAG.

O fracionamento enzimático e posterior cristalização das frações obtidas pode ser um processo para a obtenção de ácidos graxos saturados e poli-insaturados do óleo de pescado.

\section{Agradecimentos}

Ao CNPq, à CAPES e ao PET-DEPEM/SESU/MEC; aos Professores Dr. Alan Carlos Bueno e Dr. Paulo Renato Perez dos Santos (CTI-FURG); à Enga. Karen Daiane TOLOTTI e à Eng. Mayane Colpo TEIXEIRA (Lab. AIQ).

\section{Referências bibliográficas}

ANDO, Y.; SATAQUI, M.; TAKAHASHI, Y. Reinvestigation of positional distribution of fatty acids in docosahexaenoic acid-rich fish oil triacyl-sn-glycerols. Lipids, v. 35, n. 5, p. 579-582, 2000.

AUGUSTO RUIZ, W.; TORALLES, R. P. Hidrólise enzimática dos óleos vegetais. Óleos e Grãos, p. 42-48, 1999.

BERNARDINI, E. Tecnologia de Aceites y Grasas. Madrid: Ed. Alhambra, 1986. p. 229-316.

BOTTINO, N. R.; VANDENBURG, G. A.; REISER, R. Resistance of certain long-chain polyunsaturated fatty acids of marine oils to pancreatic lipase hydrolysis. Lipids, v. 2, n. 6, p. 489-493, 1967.
COntreras, E. S. G. Bioquímica de Pescados e Derivados. Jaboticabal: FUNEP, 1994. p. 315-330.

CUNHA, D. C.; PINTO, L. A. A. Estudo da etapa de fracionamento via "winterização" seca no processo de refino de óleo de pescado. Vetor, v. 12, p. 7-17. 2002

GONÇALVES, A. A.; SOUZA-SOARES, L. A. Efeitos do processamento e armazenamento na fração lípidica do pescado. Vetor, v. 10, p. 93-112, 2000.

GONÇALVES, A. A.; SOUZA-SOARES, L. A. Lipídios em peixes. Vetor, v. 8, p. 35-53, 1998.

HORROCKS, L. A.; YEO, Y. K. Health benefits of docosahexaenoic acid (DHA). Pharmacological Research, v. 40, n. 3, p. 211-225, 1999.

IKEMOTO, M.; OTA, Y. Production of two types of non-specific lipases by Geotrichum sp. FO 274A: a fish oil-assimilating strain. Journal of General and Applied Microbiology, n. 42, p. 371-379, 1996.

KINSELLA, J. E. Food components with potential therapeutic benefits: the n-3 polyunsaturated fatty acids of fish oils. Food Technology, v. 40 , n. 2, p. 89-97, 1986.

LÓPEZ-MARTÍNEZ, J. C. et al. Screening of lipases for enzymatic concentration of g-linolenic acid (GLA) from seed oils. Journal of Food Lipids, v. 13, n. 4, p. 362-374, 2006.

MAIA, E. L. Otimização da metodologia para caracterização de constituintes lípidicos e determinação da composição em ácidos graxos e aminoácidos de peixe de água doce. Campinas, 1992. Tese (Doutorado) - Universidade Estadual de Campinas - UNICAMP.

MORAES, M. M. Estudo do processo de refino do óleo de pescado. Rio Grande, 2000. 107 p. Tese (Mestrado em Engenharia de Alimentos) - Universidade Federal do Rio Grande - FURG.

MORAES, M. M.; SOUZA-SOARES, L. A. Obtenção e purificação de óleo de pescado: uma breve revisão. Vetor, v. 10, p. 81-92, 2000.

MISKANDAR, M. S. et al. Effects of Emulsifiers on crystal behavior of palm oil blends on slow crystallization. Journal of Food Lipids, v. 13, n. 1, p. 1-18, 2006.

NAMBOODIRI, V. M. H.; CHATTOPADHYAYA, R. Purification and biochemical characterization of a novel thermostable lipase from Aspergillus niger. Lipids, v. 35, n. 5, p. 495-502, 2000.

PADILHA, M. E. Obtenção de concentrado de ácidos graxos provenientes de óleo de pescado. Rio Grande, 2002. 120 p. 
Dissertação (Mestrado em Engenharia de Ciência de Alimentos) Universidade Federal do Rio Grande - FURG.

RITTNER, H. Óleo de Palma: processamento e utilização. 1 ed. São Paulo, 1996. p. 311-320.

SIMOPOULOS, A. P. Essential fatty acids in health and disease chronic disease. Food Reviews International, v. 13, n. 4, p. 623631, 1997.

STAUFFERT, D. Hidrólise Enzimática de Óleo de Pescado, Rio Grande, 2000. Dissertação (Mestrado em Engenharia de Alimentos) - Universidade Federal do Rio Grande - FURG.

TORALLES, R. P. Modelo experimental para reação de lipólise com lipase pancreática. Rio Grande, 1998. Dissertação (Mestrado em Engenharia de Alimentos) - Universidade Federal do Rio Grande - FURG.

WANASUNDARA, U. N.; SHAHIDI, F. Lipase-Assisted Concentration of n-3 Polyunsaturated Fatty Acids in Acylglycerols from Marine Oils. Journal of the American Oil Chemists' Society, v. 75, n. 8, p. 945-951, 1998.
WINDSOR, M.; BARLOW, S. Introducción a los subproductos de pesquería. Zaragoza: Acribia, 1984. p. 4-94.

$\mathrm{XU}, \mathrm{X}$. et al. Pilot batch production of specific-structured lipids by enzymatic interesterification: preliminary study on incorporation and acyl migration. Journal of the American Oil Chemists Society, v. 75 , n. 2, p. 301-308, 1998A.

XU, X. et al. Production of specific-structured lipids by enzymatic interesterification: elucidation of acyl migration by response surface design. Journal of the American Oil Chemists Society, v. 75, n. 9 , p. 1179-1186, 1998b.

YAMAGUCHI, I.; AKOH, C. C.; LAI, O. M. Modification of fish oil by Lipozyme TL IM to produce structured lipids. Journal of Foods Lipids, v. 11, n. 1, p. 65-73, 2004.

YOU, L. L.; BAHARIN, B. S. Effects of enzymatic hydrolysis on crude palm olein by lipase from Candida rugosa. Journal of Food Lipids, v. 13, n. 1, p. 73-87, 2006.

YU, F. et al. Composition of solvent-fractionated rice bran oil. Journal of Food Lipids, v. 13, p. 286-297, 2006.

ZILLER, S. Grasas y aceites alimentários. Zaragoza: Acribia, 1996. 